TRANSACTIONS OF THE

AMERICAN MATHEMATICAL SOCIETY

Volume 353, Number 10, Pages 4173-4187

S 0002-9947(01)02804-5

Article electronically published on May 17, 2001

\title{
VARIATIONAL PROBLEMS ON MULTIPLY CONNECTED THIN STRIPS III: INTEGRATION OF THE GINZBURG-LANDAU EQUATIONS OVER GRAPHS
}

\author{
JACOB RUBINSTEIN AND MICHELLE SCHATZMAN
}

\begin{abstract}
We analyze the one-dimensional Ginzburg-Landau functional of superconductivity on a planar graph. In the Euler-Lagrange equations, the equation for the phase can be integrated, provided that the order parameter does not vanish at the vertices; in this case, the minimization of the GinzburgLandau functional is equivalent to the minimization of another functional, whose unknowns are a real-valued function on the graph and a finite set of integers.
\end{abstract}

\section{INTRODUCTION}

Little and Parks discovered in 19613 that the behavior of a superconducting sample forming a long cylindrical shell is completely determined by the flux of the applied magnetic field in the axial direction. Modern experiments are performed with thin rings [4] or networks consisting of thin wires [5].

The system's behavior depends only on the geometry of the network and on the fluxes threading the finite regions bordered by the network. It is also well known that the problem is periodic with respect to each one of these fluxes.

In a previous article [7, we have shown how the Ginzburg-Landau functional in two space dimensions on a thin set can be approximated by a one-dimensional Ginzburg-Landau functional on a graph.

In this article, we shall investigate this simplified GL model from the graph theoretical viewpoint. We shall consider the GL functional on a general graph embedded in the plane. We shall partially integrate the differential equations pertaining to this model; we shall prove that the problem depends only on the fluxes of the applied magnetic field through the regions bordered by the structure.

The problem might be complicated by the zeros of the order parameter. In this article, we are only concerned with the influence of zeros on graph theoretical quantities, and we show how to integrate the phase equations also in the presence of zeros, excluding the case of zeros at the vertices of the graph. The question of existence of zeros is a delicate problem that will be addressed separately [6].

One of the interesting consequences of our analysis relates to the supercurrents. Consider for simplicity a cycle in the graph along which the order parameter does

Received by the editors March 28, 2000 and, in revised form, August 14, 2000.

2000 Mathematics Subject Classification. Primary 82D55, 49S05, 94C15, 34B45.

Key words and phrases. Graph theory, differential equations, Ginzburg-Landau functional, superconductivity.

Supported by Israel Science Foundation, CNRS, and CNRS-MOSA binational agreement.

(C)2001 American Mathematical Society 
not vanish. Clearly, the total change in the phase of the order parameter must be $2 \pi$ times some integer $N$. In the case of a single ring, one can show that $N$ is the nearest integer to $\Phi / 2 \pi$, where $\Phi$ is the flux through the hole bounded by the ring. This choice of $N$ is equivalent to minimizing the supercurrent flowing in the ring. Let us return our attention to general graphs; one might conjecture in analogy that the total phase change along a cycle in the graph divided by $2 \pi$ is the nearest integer to the flux associated to the cycle and divided by $2 \pi$. We prove in section 7 that this is not the case. The proof is based on a simple new symmetry argument.

In section 2, we introduce several graph-theoretical quantities. The GL model on the graph is described in section 3. The associated Euler-Lagrange equations are provided in section 4 and integrated in section 5 Our main result is Theorem 6.1. presented and proved in section 6 .

The classical theory of electrical networks has been largely studied from the point of view of graph theory; this theory is fundamentally different from our setup, because in our case, the currents are driven by fluxes, that are associated with the graphs, while in the electrical network theory, the currents are driven by voltage differences across edges.

\section{ElEMENTS OF GRAPH THEORY}

We recall that a combinatorial oriented finite graph $M$ is described by the data of a finite set $\mathcal{V}$ of vertices and a finite family $\mathcal{E}$ of edges, which is a list of ordered pairs of vertices; since we do not forbid loops, it is permissible to have a pair made out of two identical vertices; we do not forbid repeated edges either: this is the sense of the word family for the description of the edges. The first element of the pair of vertices in an edge is the origin and the last element is the end of the edge.

The edge-node incidence matrix of a graph $M$ is the $|\mathcal{V}| \times|\mathcal{E}|$ matrix $\mathcal{A}$ defined by its coefficients

$$
\mathcal{A}_{v j}= \begin{cases}+1 & \text { if } v \text { is the origin of the edge } j \text { and not its end } \\ -1 & \text { if } v \text { is the end of the edge } j \text { and not its origin, } \\ 0 & \text { otherwise. }\end{cases}
$$

A subgraph of $M$ is a graph $M^{\prime}$ whose vertex set $\mathcal{V}^{\prime}$ is included in the vertex set $\mathcal{V}$ of $M$, and whose edge set $\mathcal{E}^{\prime}$ is included in the edge set $\mathcal{E}$ of $M$; in particular, an edge of $M^{\prime}$ must have its extremities in $\mathcal{V}^{\prime}$.

Each edge of $M$ is numbered by $j \in\{1, \ldots,|\mathcal{E}|\}$, and each vertex is numbered by $v \in \mathcal{V}$; the number of vertices is denoted by $|\mathcal{V}|$ and the number of edges is denoted by $|\mathcal{E}|$.

A path in $M$ is a list of vertices $\left\{v_{1}, \ldots, v_{n}\right\}$ such that for each $j,\left(v_{j}, v_{j+1}\right)$ or $\left(v_{j+1}, v_{j}\right)$ belongs to the set of edges $\mathcal{E}$ of $M$. A path is elementary if all its vertices are distinct, and it is simple if all its edges are distinct. A cycle is a path whose end vertex $v_{n}$ coincides with the origin vertex $v_{1}$. It is elementary if all its vertices are distinct, except for the coincidence between the first and last vertex.

A cycle can also be seen as an algebraic sum of edges, affected with the + sign if their orientation coincides with that of the cycle, with the - sign if it is opposite and the 0 sign if the edge does not belong to the cycle; therefore, the sum of cycles can be defined, as well as the 0 cycle, and the multiplication of a cycle by any relative integer. 
Under these operations, the set of cycles of a graph $M$ is a $\mathbb{Z}$-module, and it possesses a basis of independent simple and elementary cycles. There are several such bases; a way to define one of them is the following: start from a maximal tree $T$, i.e. a maximal subgraph of $M$ without simple elementary cycles. Then adding to this subgraph any edge of $M$ which is not an edge of $T$ creates a simple elementary cycle, and the set of these cycles is precisely a basis of the cycles of $M$.

Denoting by $\mathcal{C}$ an arbitrary family of simple cycles, we may define the edge-cycle incidence matrix: it is the $|\mathcal{C}| \times|\mathcal{E}|$ matrix $\mathcal{B}(\mathcal{C}, M)$ defined by its coefficients

$$
\mathcal{B}_{l j}(\mathcal{C}, M)= \begin{cases}+1 & \text { if edge } j \text { belongs to cycle } l \text { and has the same orientation, } \\ -1 & \text { if edge } j \text { belongs to cycle } l \text { and has the opposite orientation, } \\ 0 & \text { otherwise. }\end{cases}
$$

Assume that we can embed the graph $M$ in the plane $\mathbb{R}^{2}$ : this means that the vertices are identified with points of $\mathbb{R}^{2}$ and that the edges are identified with curves of $\mathbb{R}^{2}$ parameterized by an injective mapping $\psi_{j}$ of class $C^{2}$ and of rank one from an interval $\left(a_{j}, b_{j}\right)$ to $\mathbb{R}^{2}$; we assume that $\psi_{j}, \psi_{j}^{\prime}$, and $\psi_{j}^{\prime \prime}$ can be extended as continuous bounded functions over $\left[a_{j}, b_{j}\right]$, and that $\psi_{j}^{\prime}$ is bounded away from 0 over $\left[a_{j}, b_{j}\right]$. Thus, without loss of generality, the parameter is the arc length.

It is convenient to denote by $M_{j}=\psi_{j}\left(\left(a_{j}, b_{j}\right)\right)$ the arc of $M$ indexed by $j$. We will abuse notation and identify $M$ with the union of the closed edges $\bar{M}_{j}$, $\mathcal{E}$ with the union of the edges $M_{j}$ and $\mathcal{V}$ with the union of the boundaries $\bar{M}_{j} \backslash M_{j}$. We require that for all pairs of distinct indices $j$ and $k, M_{j}$ and $\bar{M}_{k}$ have an empty intersection.

We need to describe all the arcs leaving or entering any vertex of $M$ with enough information to write Kirchhoff like transmission conditions. This is done by introducing for each $v \in \mathcal{V}$ the set $J(v)$ defined as follows:

$$
J(v)=\left\{\left(j, a_{j},+1\right): \psi_{j}\left(a_{j}\right)=v\right\} \cup\left\{\left(j, b_{j},-1\right): \psi_{j}\left(b_{j}\right)=v\right\} .
$$

If $\zeta$ belongs to $J(v)$, its components are denoted $(\zeta[1], \zeta[2], \zeta[3])$. There are $|J(v)|$ curves which start or end at any vertex $v \in \mathcal{V}$; an arc might start and end at $v$ if it is a loop.

When we consider $M$ as an embedded graph, we may define a function $u$ on it, which is equivalent to the data of the functions $u_{j}=u \circ \psi_{j}$, for $1 \leq j \leq|\mathcal{E}|$. In particular, a function $u$ belongs to the Sobolev space $H^{1}(M)$ if it is continuous over $M$ and the functions $u_{j}$ belong to $H^{1}\left(a_{j}, b_{j}\right)$ for all $j=1, \ldots,|\mathcal{E}|$.

\section{The GinzBurg-Landau MOdel}

The one-dimensional Ginzburg-Landau (GL) functional is given by the expression:

$$
\mathcal{H}(f)=\sum_{j=1}^{|\mathcal{E}|} \int_{a_{j}}^{b_{j}}\left(\left|i f_{j}^{\prime}+A_{j} f_{j}\right|^{2}+\mu\left[\left(\left|f_{j}\right|^{2}-1\right)^{2} / 2\right]\right) d \theta
$$

Here $f$ is a complex-valued order parameter, i.e. a function from $M$ to $\mathbb{C} ; A$ is a real valued function on $M$ : it is the tangential component of the magnetic vector potential $\mathbf{A}$ corresponding to the (given) applied magnetic field $\mathbf{H}_{e}$, and $\mu$ is a positive parameter which is proportional to $T_{c}-T$, where $T$ is the temperature and $T_{c}$ is the critical temperature in the absence of applied field. We require minimal 
regularity on $A$ : it suffices that for all $j \in \mathcal{E}, A_{j}=A \circ \psi_{j}$ be square integrable on $\left[a_{j}, b_{j}\right]$.

Consider a smooth fattening $\mathcal{O}(\varepsilon)$ of $M$, where $\varepsilon$ is a small positive parameter, such that the thickness of $\mathcal{O}(\varepsilon)$ away from its junctions is $2 \varepsilon$. It was shown in 7] that as $\varepsilon$ tends to 0 , the two-dimensional GL functional converges suitably to $\mathcal{H}$. Moreover, the two-dimensional supercurrents converge suitably to the currents associated with $\mathcal{H}$, and these will be defined precisely in next section.

The set $\mathbb{R}^{2} \backslash M$ has a finite number of bounded connected components which we denote by $F_{l}$; we choose the same orientation for $\mathbb{R}^{2}$ and the regions $F_{l}$; the ordered boundaries of the $F_{l}$ 's can then be identified with simple elementary cycles of $M$ : they constitute a basis of cycles of $M$ which will be denoted by $\mathcal{C}$.

The flux of the applied magnetic field $\mathbf{H}_{e}$ through $F_{l}$ is denoted by $\Phi_{l}$ : it follows from Stokes' formula that

$$
\Phi_{l}=\sum_{j \in \mathcal{E}} \mathcal{B}_{l j}(\mathcal{C}, M) \int_{a_{j}}^{b_{j}} A_{j} d \theta .
$$

The main properties of the minimizers of $\mathcal{H}$ are described by the following result 7]:

Theorem 3.1. The functional $\mathcal{H}$ has minimizers over $H^{1}(M)$; they satisfy the relation

$$
|f(\theta)| \leq 1 \quad \forall \theta \in M .
$$

Any minimizer $f$ is such that for all $j \in\{1, \ldots,|\mathcal{E}|\} f_{j}=f \circ \psi_{j}$ is of class $C^{2}$ and satisfies the Euler-Lagrange equation:

$$
\left(i \frac{d}{d \theta}+A_{j}\right)^{2} f_{j}+\mu\left(\left|f_{j}\right|^{2}-1\right) f_{j}=0, \quad \theta \in\left(a_{j}, b_{j}\right) ;
$$

at the vertices $f$ is continuous and the following Kirchhoff transmission conditions hold:

$$
\sum_{\kappa \in J(v)} \kappa[3]\left(i f_{\kappa[1]}^{\prime}+A_{\kappa[1]} f_{\kappa[1]}\right)(\kappa[2])=0 .
$$

An automorphism of $M$ is a bijection from $M$ to itself which conserves the incidence relations and the metric on the edges. We have the following corollary, which will be useful in the end of this article. Its proof is immediate.

Corollary 3.2. Let $A$ be the data in problem (3.1); denote by $\mathcal{M}(A)$ the set of global minimizers of $\mathcal{H}$ for the data $A$. Then, for any automorphism $\sigma$ of $M$, the set $\mathcal{M}(A \circ \sigma)$ of minimizers of $\mathcal{H}$ for the data $A \circ \sigma$ is the image of $\mathcal{M}(A)$ by the mapping $f \mapsto f \circ \sigma$.

\section{The Euler-Lagrange equations for the one-Dimensional GinZBURG-LANDAU EQUATION}

The minimization of $\mathcal{H}$ concerns a complex valued function $f$ on $M$ and the data are given by the auxiliary function $A$. It is therefore remarkable that it can be reduced in many cases to a minimization problem involving a scalar function $y$ on $M$ and a finite number of integers; the data will be a finite set of real numbers. 
The main idea is that we will be able to integrate the phase equation; however, zeros of the order parameter will give us trouble, and they will increase the complexity of the functional that we construct.

Let us start by a simple functional analytic observation. If a function $z$ belongs to $H^{1}\left(\left(a_{j}, b_{j}\right) ; \mathbb{C}\right)$, it vanishes at some point iff

$$
\int_{a_{j}}^{b_{j}} \frac{d \theta}{|z(\theta)|^{2}}=+\infty
$$

Indeed, if $f$ does not vanish over the compact set $\left[a_{j}, b_{j}\right]$, then since $z$ belongs to a set of continuous functions, $|z|$ is bounded away from 0 , and thus, the integral in (4.1) is bounded; conversely, assume that $z$ vanishes at some point; if $z$ is identically equal to 0 , the conclusion is clear; if $z$ is not identically equal to 0 , let $\left(c^{\prime}, c^{\prime \prime}\right)$ be a connected component of the set $\left(a_{j}, b_{j}\right) \backslash z^{-1}(\{0\})$; if for instance $z\left(c^{\prime}\right)$ vanishes, we observe that a continuous determination of $\log z(\theta)$ can be defined over $\left(c^{\prime}, c^{\prime \prime}\right)$ and that

$$
\lim _{\theta \downarrow c^{\prime}}|\ln z(\theta)|=+\infty
$$

but

$$
\frac{d}{d \theta} \ln z(\theta)=\frac{z^{\prime}(\theta)}{z(\theta)}
$$

and therefore, for any $c \in\left(c^{\prime}, c^{\prime \prime}\right)$ and all $\theta \in\left(c^{\prime}, c\right)$ :

$$
|\ln z(c)-\ln z(\theta)| \leq \int_{\theta}^{c}\left|\frac{z^{\prime}(\theta)}{z(\theta)}\right| d \theta \leq\left(\int_{\theta}^{c}\left|z^{\prime}(\theta)\right|^{2} d \theta\right)^{1 / 2}\left(\int_{\theta}^{c} \frac{d \theta}{|z(\theta)|^{2}}\right)^{1 / 2}
$$

Since $f_{j}^{\prime}$ is square integrable, the second integral on the right hand side of (4.2) must tend to infinity as $\theta$ decreases to $c^{\prime}$.

In what follows, $f$ is a minimizer of $\mathcal{H}$ over $H^{1}(M ; \mathbb{C})$. On each connected component of $M \backslash f^{-1}(\{0\})$, there is a determination $\phi$ of the argument of $f /|f|$ such that $\exp (i \phi)$ is a continuous function over $M$.

Furthermore, on each connected component of $\left[a_{j}, b_{j}\right] \backslash f_{j}^{-1}(0), y_{j}=\left|f_{j}\right|$ and $\phi_{j}$ satisfy the following pair of differential equations, obtained by taking the real part and the imaginary part of (3.4):

$$
\begin{aligned}
& -y_{j}^{\prime \prime}+\left(A_{j}-\phi_{j}^{\prime}\right)^{2} y_{j}+\mu y_{j}\left(y_{j}^{2}-1\right)=0, \\
& \left(A_{j}-\phi_{j}^{\prime}\right) y_{j}^{\prime}+\left(A_{j}-\phi_{j}^{\prime}\right)^{2} y_{j}=0 .
\end{aligned}
$$

Multiply equation (4.4) by $y_{j}$ and integrate: we obtain

$$
\left(\phi_{j}^{\prime}-A_{j}\right) y_{j}^{2}=\text { constant. }
$$

The constant on the right hand side of (4.5) vanishes iff $y_{j}$ vanishes somewhere on $\left[a_{j}, b_{j}\right]$. We have seen in [7] that this constant is the branch current $I_{j}$. Thus, we write more explicitly

$$
\left(\phi_{j}^{\prime}-A_{j}\right) y_{j}^{2}=I_{j} .
$$


At each vertex where $f$ does not vanish, condition (3.5) is equivalent to the two conditions

$$
\begin{aligned}
& \sum_{\kappa \in J(v)} \kappa[3] y_{\kappa[1]}^{\prime}(\kappa[2])=0, \\
& \sum_{\kappa \in J(v)} \kappa[3] I_{\kappa[1]}=0 .
\end{aligned}
$$

If $I_{j}$ does not vanish, we have the relation

$$
\int_{a_{j}}^{b_{j}}\left(\phi_{j}^{\prime}-A_{j}\right) d \theta=I_{j} \int_{a_{j}}^{b_{j}} \frac{d \theta}{y_{j}^{2}}
$$

Thanks to standard results in the theory of ordinary differential equations, if $f_{j}$ vanishes on $\left(a_{j}, b_{j}\right)$, either it vanishes identically, or it vanishes at isolated points. If $f$ vanishes at an isolated point $\theta_{0}$ of $\left[a_{j}, b_{j}\right]$, then, by uniqueness of solutions of ordinary differential equations, $f_{j}^{\prime}\left(\theta_{0}\right)$ cannot vanish; therefore, thanks to the Taylor expansion

$$
f_{j}\left(\theta_{0}\right)=f_{j}^{\prime}\left(\theta_{0}\right)\left(\theta-\theta_{0}\right)+\text { higher order terms, }
$$

we must have the identity

$$
y_{j}^{\prime}\left(\theta_{0}+0\right)+y_{j}^{\prime}\left(\theta_{0}-0\right)=0 .
$$

Hence, any determination of the phase jumps by an odd number times $\pi$ at the zeros of $f_{j}$; therefore, if $p_{j}$ is the number of zeros of $f_{j}$ over the open subset $\left(a_{j}, b_{j}\right)$, we combine this information with (4.6), and we obtain

$$
\phi_{j}\left(b_{j}\right)-\phi_{j}\left(a_{j}\right)=p_{j} \pi+\int_{a_{j}}^{b_{j}} A_{j}(s) d s \bmod 2 \pi,
$$

provided that $f_{j}$ does not vanish at $a_{j}$ or $b_{j}$.

\section{Integration of the Phase Equations}

We assume henceforth that $f$ is a minimizer of $\mathcal{H}$ which does not vanish at the vertices of the graph $M$. Under this assumption, we will obtain another representation of the one-dimensional Ginzburg-Landau functional, which will involve graph theoretical objects, such as the incidence matrices defined in section 2.

Any cycle $m$ of $M$ is a linear combination of the $\partial F_{l}$ 's, which are the oriented boundaries of the $F_{l}$ 's:

$$
m=\sum_{l=1}^{|\mathcal{C}|} \epsilon_{m l} \partial F_{l}
$$

with coefficients $\epsilon_{m l} \in \mathbb{Z}$; thus we can define for every cycle $m$ a flux $\Phi_{m}$ through the oriented region bounded by this cycle through

$$
\Phi_{m}=\sum_{l=1}^{|\mathcal{C}|} \epsilon_{l m} \Phi_{l} .
$$

Define $\widehat{M}$ to be the subgraph of $M$ whose vertex set is $\mathcal{V}$, and whose edges are precisely those edges of $M$ on which the branch current does not vanish. The edge-node incidence matrix for $\widehat{M}$ is the matrix $\widehat{\mathcal{A}}$ obtained by erasing from $\mathcal{A}$ the columns indexed by $j \notin \widehat{\mathcal{E}}$. 
It should be observed that at least two edges are incident at all vertices of $\widehat{M}$; for if there existed a vertex $v$ with an unique incident edge $j$, then relation (4.8) would imply that $I_{j}$ vanishes, a contradiction. In particular, for all edge $j$ of $\widehat{M}$ there exists a cycle $m$ containing the edge $j$ with a non-vanishing coefficient.

Let $\widehat{\mathcal{C}}$ be a basis of simple and elementary cycles of $\widehat{M}: f$ does not vanish along any cycle $m \in \widehat{\mathcal{C}}$; thus the expression

$$
\sum_{j \in \widehat{\mathcal{E}}} \mathcal{B}_{m j}(\widehat{\mathcal{C}}, \widehat{M}) \int_{a_{j}}^{b_{j}} \phi_{j}^{\prime} d \theta
$$

is well defined, and it is the phase difference along a cycle $m \in \widehat{\mathcal{C}}$. As $f$ is a continuous function, the expression (5.1) must be a multiple of $2 \pi$, which we denote by $2 \pi N_{m}$ :

$$
\sum_{j \in \widehat{\mathcal{E}}} \mathcal{B}_{m j}(\widehat{\mathcal{C}}, \widehat{M}) \int_{a_{j}}^{b_{j}} \phi_{j}^{\prime} d \theta=2 \pi N_{m} .
$$

Define now for each real-valued measurable function $z$ on $\left(a_{j}, b_{j}\right)$

$$
\Lambda_{j}(z)= \begin{cases}\int_{a_{j}}^{b_{j}} z^{-2} d \theta & \text { if } z^{-2} \text { is integrable } \\ +\infty & \text { otherwise }\end{cases}
$$

For all measurable functions $y$ on $M$, let $\Lambda(y)$ be the $|\mathcal{E}| \times|\mathcal{E}|$ diagonal matrix

$$
\Lambda_{i j}(y)=\delta_{i j} \Lambda_{j}\left(y_{j}\right) .
$$

For all $m \in \widehat{\mathcal{C}}$, we multiply (4.9) by $\mathcal{B}_{m j}(\widehat{\mathcal{C}}, \widehat{M})$, and we sum with respect to $j \in \mathcal{E}$; with the help of relations (3.2), (5.2) and (5.4), we see that

$$
2 \pi N_{m}=\Phi_{m}+(\mathcal{B}(\widehat{\mathcal{C}}, \widehat{M}) \Lambda I)_{m} .
$$

We now give an alternative formulation of this expression. Observe that the above defined basis $\widehat{\mathcal{C}}$ of the cycle space of $\widehat{M}$ consists of cycles of $M$; let $\mathcal{B}(\widehat{\mathcal{C}}, M)$ be the edge-cycle incidence matrix for $\widehat{\mathcal{C}}$ in $M$; then the edge-cycle incidence matrix $\mathcal{B}(\widehat{\mathcal{C}}, \widehat{M})$ is simply obtained by erasing from $\mathcal{B}(\widehat{\mathcal{C}}, M)$ all the columns indexed by $j \notin \widehat{\mathcal{E}}$.

Define further the $|\widehat{\mathcal{E}}| \times|\widehat{\mathcal{E}}|$ matrix $\widehat{\Lambda}(y)$ by

$$
(\widehat{\Lambda}(y))_{j k}=\delta_{j k} \Lambda_{j}(y), \quad \forall j, k \in \widehat{\mathcal{E}}
$$

We write

$$
\widehat{I}=\left(I_{j}\right)_{j \in \widehat{\mathcal{E}}}, \quad \widehat{\xi}=2 \pi \widehat{N}-\widehat{\Phi}=\left(2 \pi N_{m}-\Phi_{m}\right)_{m \in \widehat{\mathcal{C}}} .
$$

With notations (5.6), relation (5.5) can be rewritten as

$$
\widehat{\xi}=\widehat{\mathcal{B}} \widehat{\Lambda}(y) \widehat{I} .
$$

We observe that if the edge $j$ is a loop starting and ending at $v$, the terms containing $I_{j}$ cancel out in 4.8); there are no contributions of $\mathcal{E} \backslash \widehat{\mathcal{E}}$ to (4.8). Thus relation (4.8) can be rewritten

$$
\forall v \in \mathcal{V}, \quad \sum_{j \in \widehat{\mathcal{E}}} \widehat{\mathcal{A}}_{v j} \widehat{I}_{j}=0
$$


or in a more concise form

$$
\widehat{\mathcal{A}} \widehat{I}=0 .
$$

Now, we show how to take into account the edges where the branch current vanishes. Indeed, if $m$ is a cycle, we denote by $Z_{m}(f)$ the number of zeros along this cycle, counted algebraically. Then, since, according to (4.10) and (4.9)

$$
\int_{a_{j}}^{b_{j}} \phi_{j}^{\prime} d \theta= \begin{cases}\Lambda_{j} I_{j}+\int_{a_{j}}^{b_{j}} A_{j}(s) d s & \text { if } I_{j} \neq 0, \\ p_{j} \pi+\int_{a_{j}}^{b_{j}} A_{j}(s) d s & \text { if } I_{j}=0\end{cases}
$$

we see that (5.5) generalizes as

$$
2 \pi N_{m}=(\mathcal{B} \Lambda I)_{m}+\Phi_{m}+Z_{m}(f) \pi .
$$

We state first a graph-theoretical lemma which gives the properties of the system (5.7), (5.8)

Lemma 5.1. For all choices of $\widehat{\mathcal{E}} \subset \mathcal{E}$ and for all diagonal $|\widehat{\mathcal{E}}| \times|\widehat{\mathcal{E}}|$ matrices $\widehat{\Lambda}$ with strictly positive diagonal terms, there exists a unique solution of the system (5.8), (5.7); moreover, this solution is given by

$$
\widehat{I}=\widehat{\mathcal{B}}^{T}\left(\widehat{\mathcal{B}} \widehat{\Lambda}(y) \widehat{\mathcal{B}}^{T}\right)^{-1} \widehat{\xi} .
$$

Proof. It is a classical result of graph theory (see for instance [1]) that if $\widehat{\mathcal{A}} \widehat{I}=0$, there exists a vector $J \in \mathbb{R}^{|\widehat{\mathcal{C}}|}$ such that

$$
I=\widehat{\mathcal{B}}^{T} J
$$

We substitute this relation into (5.7), and we find that we have to solve

$$
\widehat{\mathcal{B}} \widehat{\Lambda}(y) \widehat{\mathcal{B}}^{T} J=\widehat{\xi} .
$$

It is immediate that $\operatorname{ker} \widehat{\mathcal{B}}^{T}=\operatorname{ker}\left(\widehat{\mathcal{B}} \widehat{\Lambda}(y) \widehat{\mathcal{B}}^{T}\right)$. It is also a classical result of graph theory that $\widehat{\mathcal{B}}$ is of maximal rank; since there are more edges than cycles, the rank of $\widehat{\mathcal{B}}$ is $|\widehat{\mathcal{C}}|$. Therefore the kernel of $\widehat{\mathcal{B}}^{T}$ is reduced to $\{0\}$ and the result follows.

This lemma enables us to give an expression of $\mathcal{H}(f)$ in terms of $y$ : we have

$$
\left|i f_{j}^{\prime}+A_{j} f_{j}\right|^{2}=\left|y_{j}^{\prime}\right|^{2}+y_{j}^{2}\left(\phi_{j}^{\prime}-A_{j}\right)^{2}
$$

and thanks to (4.6),

$$
\left|i f_{j}^{\prime}+A_{j} f_{j}\right|^{2}=\left|y_{j}^{\prime}\right|^{2}+I_{j}^{2} y_{j}^{-2} .
$$

Therefore, we obtain the identity

$$
\sum_{j \in \mathcal{E}} \int_{a_{j}}^{b_{j}}\left|i f_{j}^{\prime}+A_{j} f_{j}\right|^{2} d \theta=\sum_{j \in \mathcal{E}} \int_{a_{j}}^{b_{j}}\left|y_{j}^{\prime}\right|^{2} d \theta+\sum_{j \in \widehat{\mathcal{E}}} \Lambda_{j}(y) I_{j}^{2} .
$$

We infer from Lemma 5.1 that

$$
\sum_{j \in \widehat{\mathcal{E}}} I_{j}^{2} \Lambda_{j}(y)=\widehat{\xi}^{T}\left(\widehat{\mathcal{B}} \widehat{\Lambda}(y) \widehat{\mathcal{B}}^{T}\right)^{-1} \widehat{\xi}
$$

Thus we have proved the following result: 
Lemma 5.2. Let $f$ be a minimizer of $\mathcal{H}$ over $H^{1}(M)$ which does not vanish at the vertices of $M$, and let $y=|f|$; let $\widehat{\mathcal{E}}$ be the set of edge indices for which the branch current $I_{j}$ does not vanish and let $\widehat{\mathcal{C}}$ be a maximal set of independent cycles of $M$ on which the branch current does not vanish. Then there exists an integer vector $\widehat{N} \in \mathbb{Z}^{\widehat{c}}$, such that

$$
\mathcal{H}(f)=\sum_{j \in \mathcal{E}} \int_{a_{j}}^{b_{j}}\left[\left|y_{j}^{\prime}\right|^{2}+\mu\left(y_{j}^{2}-1\right)^{2} d \theta+(2 \pi \widehat{N}-\widehat{\Phi})^{T}\left(\widehat{\mathcal{B}} \widehat{\Lambda}(y) \widehat{\mathcal{B}}^{T}\right)^{-1}(2 \pi \widehat{N}-\widehat{\Phi})^{T} .\right.
$$

We will now extend the previous formulation so as to include the case of vanishing branch currents. In particular, we will show that $\xi^{T}\left(\mathcal{B} \Lambda \mathcal{B}^{T}\right)^{-1} \xi$, which is defined for all diagonal matrices $\Lambda$ with strictly positive diagonal terms can be extended by continuity to diagonal matrices $\Lambda$ with some infinite diagonal terms; basically, we take a sequence of matrices $\Lambda_{n}$ with some fixed diagonal terms and the remaining diagonal terms tending to infinity, and we prove that the limit of $\xi^{T}\left(\mathcal{B} \Lambda_{n} \mathcal{B}^{T}\right)^{-1} \xi$ is well defined and is independent of the particular limiting process that we have chosen.

We also give a graph theoretical interpretation of the limiting expression. The foregoing result is in the same spirit as the work [2] of Colin de Verdière, Pan and Ycart, whose influence we acknowledge here.

Lemma 5.3. Let $\widetilde{\mathcal{E}}$ be a subset of $\mathcal{E}$ and let $\Lambda_{n}$ be a sequence of diagonal matrices such that

$$
\forall j \in \widetilde{\mathcal{E}}, \quad 0<\left(\Lambda_{n}\right)_{j j} \rightarrow \infty ; \quad \forall j \notin \widetilde{\mathcal{E}}, \quad\left(\Lambda_{n}\right)_{j j}=\Lambda_{j}>0 .
$$

Then the limit of the expression $\xi^{T}\left(\mathcal{B} \Lambda_{n} \mathcal{B}^{T}\right)^{-1} \xi$ as $n$ tends to infinity exists. Moreover we can characterize this limit as follows: there exists a basis $\overline{\mathcal{C}}$ of cycles of $M$ and a renumbering of the edges such that the edge-cycle incidence matrix $\overline{\mathcal{B}}=\mathcal{B}(\overline{\mathcal{C}}, M)$ is equal to

$$
\overline{\mathcal{B}}=\left(\begin{array}{cc}
\overline{\mathcal{B}}_{11} & \overline{\mathcal{B}}_{12} \\
0 & \overline{\mathcal{B}}_{22}
\end{array}\right)
$$

The renumbering of the edges sends the indices of $\widetilde{\mathcal{E}}$ to $\{1, \ldots,|\widetilde{\mathcal{E}}|\}$. Write $\overline{\mathcal{B}}=$ $S \mathcal{B} P_{\sigma}$ with $P_{\sigma}$ a permutation matrix and $S$ a row operation matrix: for row operations we consider only multiplications of rows by \pm 1 and subtraction or addition to any row of any other row; let $P^{(2)}$ be the projection on the space of rows of $\overline{\mathcal{B}}_{22}$ and let $\Lambda^{(2)}$ be the restriction of $P_{\sigma}^{T} \Lambda_{n} P_{\sigma}$ to the space of columns of $\overline{\mathcal{B}}_{22}$; then

$$
\lim _{n \rightarrow \infty} \xi^{T}\left(\mathcal{B} \Lambda_{n} \mathcal{B}^{T}\right)^{-1} \xi=\xi^{T} S^{-1} P^{(2)}\left(\overline{\mathcal{B}}_{22} \Lambda^{(2)} \overline{\mathcal{B}}_{22}^{T}\right)^{-1} P^{(2)} S^{-T} \xi .
$$

Proof. Let $E$ be the edge space of $M$, i.e.

$$
E=\mathbb{R}^{\mathcal{E}}
$$

in other words, $E$ is the space of formal combinations of edges of $M$. The space $E$ will be equipped with the canonical scalar product of $\mathbb{R}^{\mathcal{E}}$.

The subspace $\widetilde{E}$ is the space of formal combinations of edges in $\widetilde{\mathcal{E}}$ : it is a subspace of $E$ of dimension $|\widetilde{\mathcal{E}}|$. The complementary set of $\widetilde{\mathcal{E}}$ in $\mathcal{E}$ is called $\widehat{\mathcal{E}}$, and the space of formal linear combinations of edges belonging to $\widehat{\mathcal{E}}$ is the space $\widehat{E}$ which is orthogonal to $\widetilde{E}$. 
Denote by $\widetilde{Q}$ the orthogonal projection in $E$ whose image is $\widetilde{E}$, and let us prove that the kernel of $\mathcal{B} \Lambda_{n} \widetilde{Q} \mathcal{B}^{T}$ coincides with the kernel of $\mathcal{B} \widetilde{Q} \mathcal{B}^{T}$. Since $\Lambda_{n}$ and $\widetilde{Q}$ commute, these two operators are self-adjoint in $E$, and thus it is equivalent to prove that the kernel of $\mathcal{B} \Lambda_{n} \widetilde{Q} \mathcal{B}^{T}$ is identical to the kernel of $\mathcal{B} \widetilde{Q} \mathcal{B}^{T}$. Let indeed $z$ belong to the kernel of $\mathcal{B} \widetilde{Q} \mathcal{B}^{T}$ :

$$
\mathcal{B} \widetilde{Q} \mathcal{B}^{T} z=0
$$

we multiply this relation by $z^{T}$ on the left; thanks to the properties of projections we find that

$$
\left|\widetilde{Q} \mathcal{B}^{T} z\right|=0
$$

which means that $\mathcal{B}^{T} z$ belongs to the kernel of $\widetilde{Q}$, i.e. $\mathcal{B}^{T} z$ belongs to $\widehat{E}=\operatorname{ker} \widetilde{Q}$. It is then immediate that $\mathcal{B} \Lambda_{n} \widetilde{Q} \mathcal{B}^{T} z$ vanishes. Conversely, if

$$
\mathcal{B} \Lambda_{n} \widetilde{Q} \mathcal{B}^{T} z=0,
$$

we multiply this relation on the left by $z^{T}$; since $\Lambda_{n}$ and $\widetilde{Q}$ commute, we find that

$$
\Lambda_{n}^{1 / 2} \widetilde{Q} \mathcal{B}^{T} z=0
$$

Since $\Lambda_{n}$ is invertible in $E$, we can see that $\widetilde{Q} \mathcal{B} z$ vanishes, and our contention is proved.

Define the following numbers:

$$
\lambda_{n}=\min _{j \in \widetilde{\mathcal{E}}}\left(\Lambda_{n}\right)_{j j}, \quad \lambda=\min _{n \in \mathbb{N}, j \in \mathcal{E}}\left(\Lambda_{n}\right)_{j j} .
$$

Denote by $\widetilde{P}$ the orthogonal projection on the image of $\mathcal{B} \widetilde{Q} \mathcal{B}^{T}$; there exists a number $\mu>0$ such that for all $z \in E$ :

$$
z^{T} \mathcal{B} \widetilde{Q} \mathcal{B}^{T} z \geq \mu z^{T} \widetilde{P} z
$$

We infer from (5.11) that we have also the estimate

$$
\forall z \in E, \quad z^{T} \mathcal{B} \Lambda_{n} \widetilde{Q} \mathcal{B}^{T} z \geq \mu \lambda_{n} z^{T} \widetilde{P} z .
$$

Since $\mathcal{B}$ has more columns than rows and is of maximal rank, $\mathcal{B B}^{T}$ is invertible; if we denote the norm of its inverse by $\nu^{-1}$, we have the estimate:

$$
\forall z \in E, \quad z^{T} \mathcal{B B}^{T} z \geq \nu|z|^{2} .
$$

Thus, for all $n$ large enough, we infer from (5.11) that

$$
z^{T} \mathcal{B} \Lambda_{n} \mathcal{B}^{T} z \geq \lambda \nu|z|^{2} \text {. }
$$

Consider the system

$$
\mathcal{B} \Lambda_{n} \mathcal{B}^{T} \alpha_{n}=\xi
$$

If we multiply (5.14) by $\alpha_{n}^{T}$ on the left we obtain the identity

$$
\alpha_{n}^{T} \mathcal{B} \Lambda_{n} \mathcal{B}^{T} \alpha_{n}=\alpha_{n}^{T} \xi
$$

and we deduce from (5.13) that

$$
\left|\alpha_{n}\right| \leq|\xi| /(\lambda \nu)
$$

On the other hand, identity (5.15) together with (5.12) and (5.16) yield

$$
\lambda_{n} \mu\left|\widetilde{P} \alpha_{n}\right|^{2} \leq|\xi|^{2}(\lambda \nu) .
$$


Therefore, $\widetilde{P} \alpha_{n}$ tends to 0 as $n$ tends to infinity.

Apply now $1-\widetilde{P}$ to equation (5.14): by definition of $\widetilde{P}$, we have the identity

$$
(\mathbf{1}-\widetilde{P}) \mathcal{B} \Lambda_{n} \widetilde{Q} \mathcal{B}^{T}(\mathbf{1}-\widetilde{P})=0
$$

thus we obtain

$$
(\mathbf{1}-\widetilde{P}) \mathcal{B} \Lambda_{n}(\mathbf{1}-\widetilde{Q}) \mathcal{B}^{T}(\mathbf{1}-\widetilde{P}) \alpha_{n}=(\mathbf{1}-\widetilde{P}) \xi-(\mathbf{1}-\widetilde{P}) \mathcal{B} \Lambda_{n} \mathcal{B}^{T} \widetilde{P} \alpha_{n} .
$$

The right hand side of equation (5.17) tends to $(\mathbf{1}-\widetilde{P}) \xi$ as $n$ tends to infinity; on the left hand side, the operator $(\mathbf{1}-\widetilde{P}) \mathcal{B} \Lambda_{n}(\mathbf{1}-\widetilde{Q}) \mathcal{B}^{T}(\mathbf{1}-\widetilde{P})$ does not depend on $n$, according to assumption (5.10). If we call $L$ its restriction to the space ker $\widetilde{P}$, observe that it is an operator of full rank, since it is a diagonal block of the block decomposition of $\mathcal{B} \Lambda_{n} \mathcal{B}^{T}$ along $\operatorname{Im} \widetilde{P} \oplus \operatorname{Im}(1-\widetilde{P})$. Therefore $L$ is invertible and $\alpha_{n}$ tends to $L^{-1}(\mathbf{1}-\widetilde{P}) \xi$ as $n$ tends to infinity.

We can see now that the limit of $\alpha_{n}^{T} \mathcal{B} \Lambda_{n} \mathcal{B}^{T} \alpha_{n}$ exists and is equal to $\xi^{T}(\mathbf{1}-\widetilde{P}) L^{-1}(\mathbf{1}-\widetilde{P}) \xi$.

There remains to give a graph theoretical description of the operators $L$ and $\widetilde{P}$ to complete the proof of the lemma.

Let $\widehat{M}$ be the graph whose vertex set is $\mathcal{V}$ and whose edge family is $\widehat{\mathcal{E}}$. It is possible to find a basis of cycles $\widehat{\mathcal{C}}$ of $\widehat{M}$, and to complete this into a basis of cycles of $M$ by adding to it a set $\widetilde{\mathcal{C}}$. The resulting basis of cycles will be $\overline{\mathcal{C}}=\widetilde{\mathcal{C}} \cup \widehat{\mathcal{C}}$, and we order it in such a way that the elements of $\widetilde{\mathcal{C}}$ come first. We also choose an ordering of the edges in such a way that the elements of $\widetilde{\mathcal{E}}$ also come first. Let now $\overline{\mathcal{B}}=\mathcal{B}(\overline{\mathcal{C}}, M)$ be written in the block form

$$
\overline{\mathcal{B}}=\left(\begin{array}{ll}
\overline{\mathcal{B}}_{11} & \overline{\mathcal{B}}_{12} \\
\overline{\mathcal{B}}_{21} & \overline{\mathcal{B}}_{22}
\end{array}\right)
$$

Under our assumptions, the block $\overline{\mathcal{B}}_{21}$ vanishes. The matrix $\mathcal{B}$ is related to the matrix $\overline{\mathcal{B}}$ by a transformation of the form

$$
\overline{\mathcal{B}}=S \mathcal{B} P_{\sigma}
$$

where $S$ is a row operation matrix and $P_{\sigma}$ a permutation matrix.

We set up a block decomposition of $\mathbb{R}^{\mathcal{E}}$ into $\mathbb{R}^{\tilde{\mathcal{E}}} \oplus \mathbb{R}^{\widehat{\mathcal{E}}}$ and of $\mathbb{R}^{\overline{\mathcal{C}}}$ into $\mathbb{R}^{\widetilde{\mathcal{C}}} \oplus \mathbb{R}^{\widehat{\mathcal{C}}}$. We let $\Lambda_{n}^{(1)}$ be the block of $P_{\sigma}^{T} \Lambda_{n} P_{\sigma}$ relative to the first $|\widetilde{\mathcal{E}}|$ lines and $\Lambda^{(2)}$ be the block of that matrix relative to the last $|\widehat{\mathcal{E}}|$ lines; our construction ensures that $P_{\sigma}^{T} \Lambda P_{\sigma}$ is block diagonal. Then $\mathcal{B} \Lambda_{n} \mathcal{B}^{T}$ can be rewritten as follows:

$$
S^{-1}\left(\begin{array}{cc}
\overline{\mathcal{B}}_{11} \Lambda_{n}^{(1)} \overline{\mathcal{B}}_{11}^{T}+\overline{\mathcal{B}}_{12} \Lambda^{(2)} \overline{\mathcal{B}}_{12}^{T} & \overline{\mathcal{B}}_{12} \Lambda^{(2)} \overline{\mathcal{B}}_{22}^{T} \\
\overline{\mathcal{B}}_{22} \Lambda^{(2)} \overline{\mathcal{B}}_{12}^{T} & \overline{\mathcal{B}}_{22} \Lambda^{(2)} \overline{\mathcal{B}}_{22}^{T}
\end{array}\right) S^{-T} .
$$

It is clear on the form of this matrix that the limit of $\xi^{T}\left(\mathcal{B} \Lambda_{n} \mathcal{B}^{T}\right)^{-1} \xi$ as $n$ tends to infinity is

$$
\xi^{T} S^{-1} P^{(2)}\left(\overline{\mathcal{B}}_{22} \Lambda^{(2)} \overline{\mathcal{B}}_{22}^{T}\right)^{-1} P^{(2)} S^{-T} \xi
$$

where $P^{(2)}$ is the orthogonal projection on the last $|\widehat{\mathcal{E}}|$ component of a vector. Thus, we have identified the limit as stated. 


\section{The REDUCED FUnCTIONAL}

We introduce now the functional

$$
\mathcal{K}(y, \xi, \Lambda)=\sum_{j \in \mathcal{E}} \int_{a_{j}}^{b_{j}}\left(\left|y_{j}^{\prime}\right|^{2}+\mu\left(y_{j}^{2}-1\right)^{2} / 2\right) d \theta+(\xi)^{T}\left(\mathcal{B} \Lambda \mathcal{B}^{T}\right)^{-1}(\xi),
$$

where $\Lambda$ is a diagonal matrix with diagonal entries belonging to $(0,+\infty]$, and the expression $\xi^{T}\left(\mathcal{B} \Lambda \mathcal{B}^{T}\right)^{-1} \xi$ is defined by the passage to the limit in Lemma 5.3.

We can state and prove now the main result of this section, recalling that $Z_{m}(y)$ is the number of zeros of $y$ along the cycle $m$.

Theorem 6.1. Let $\Lambda(y)$ be defined by (5.3) and (5.4). Then

$$
\begin{aligned}
& \min \left\{\mathcal{H}(f), f \in H^{1}(M ; \mathbb{C})\right\} \\
& \quad=\min \left\{\mathcal{K}(y, \xi+\pi Z(y), \Lambda(y)), y \in H^{1}(M), \xi \in 2 \pi \mathbb{Z}^{|\mathcal{C}|}-\Phi\right\} .
\end{aligned}
$$

Proof. Assume that $f$ is a minimizer of $\mathcal{H}$; as a consequence of Lemmas 5.1 and 5.2, and of relation (5.9) we see that for $y=|f|$ and $\xi=2 \pi N-\Phi$,

$$
\mathcal{H}(f)=\mathcal{K}(y, \xi+\pi Z(y), \Lambda(y)) .
$$

Assume now that $(y, \xi)$ is a minimizer of $\mathcal{K}(y, \xi+\pi Z(y), \Lambda(y))$ over the set $\left\{(y, \xi): y \in H^{1}(M), \xi \in \Phi-2 \pi \mathbb{Z}^{|\mathcal{C}|}\right\}$, and that $y$ does not vanish at the vertices of $M$.

Without loss of generality, we may assume that $y$ is nonnegative, since

$$
\mathcal{K}(y, \xi+\pi Z(y), \Lambda(y))=\mathcal{K}(|y|, \xi+\pi Z(|y|), \Lambda(|y|)) .
$$

Let $\widehat{M}$ be the subgraph of $M$ whose vertex set is $\mathcal{V}$ and whose edge set is precisely those edges on which $y$ does not vanish. Let $\widehat{C}$ and $\mathcal{B}(\widehat{C}, \widehat{M})=\widehat{\mathcal{B}}$ be exactly as in section 5 then the branch currents are defined by

$$
I_{\widehat{\mathcal{E}}}=\widehat{\mathcal{B}}^{T}\left(\widehat{\mathcal{B}} \widehat{\Lambda}(y) \widehat{\mathcal{B}}^{T}\right)^{-1} \widehat{\xi},
$$

and

$$
I_{\mathcal{E} \backslash \widehat{\mathcal{E}}}=0 .
$$

Pick an arbitrary vertex of $M$, and let the phase vanish at this particular point. On any edge of the graph, define the phase differences by

$$
\phi(\theta)-\phi\left(a_{j}\right)= \begin{cases}\int_{a_{j}}^{\theta}\left(A_{j}+\frac{I_{j}}{y_{j}^{2}}\right) d \tau & \text { if } j \in \widehat{\mathcal{E}}, \\ \int_{a_{j}}^{\theta}\left(A_{j}+\pi \sum_{i=1}^{p_{j}} \delta\left(\tau-z_{j, i}\right)\right) d \tau & \text { otherwise. }\end{cases}
$$

where $z_{j, 1}, \ldots, z_{j, p_{j}}$ are the zeros of $y$ along the branch $j$ with $j \notin \widehat{\mathcal{E}}$.

The reader will check that this construction of the phase is consistent, so that $f=y \exp (i \phi)$ is a continuous function which satisfies $\mathcal{H}(f)=\mathcal{K}(y, \xi+\pi Z(y), \Lambda(y))$.

Since we have obtained a value $\mathcal{H}(f)$ which is at least equal to the minimum of the values of $\mathcal{H}$, we have proved the desired identities. 
We were able to integrate the phase equations up to a set of integers $\left\{N_{l}, l=\right.$ $1, \ldots,|\mathcal{C}|\}$. From the definition of $\xi_{l}$ and $(\underline{6.1})$ we draw the important conclusion that the minimizers are periodic with respect to each $\Phi_{l}$ with a (non-dimensional) period of $2 \pi$; more precisely, if we assign to each vector of data $\Phi$ a set of minimizers, i.e. a set of pairs $(y, \xi)$, we get exactly the same set of minimizers when we add to the data an integer vector multiplied by $2 \pi$. In the case of a single ring this was confirmed experimentally by Little and Parks [3. If the matrix $\left(\mathcal{B}^{T} \Lambda \mathcal{B}\right)^{-1}$ is diagonal, then when there are no zeros, for any value of $\Lambda$ the energy is minimized when every $\xi_{l}^{2}$ is minimized. Therefore each $N_{l}$ is the closest integer to $\Phi_{l} / 2 \pi$. This is the case, for example, when every edge of $M$ is a closed loop. For arbitrary $M$, however, the determination of the $\left\{N_{l}\right\}$ cannot be decoupled from the amplitude equations, which we now rewrite as

$$
y_{j}^{\prime \prime}+y_{j}^{-3} I_{j}^{2}+\mu y_{j}\left(y_{j}^{2}-1\right)=0, \quad j=1,2, \ldots,|\mathcal{E}|,
$$

together with the Kirchhoff conditions

$$
\sum_{\kappa \in J(v)} \kappa[3] y_{\kappa[1]}^{\prime}(\kappa[2])=0
$$

for all $v \in \mathcal{V}$.

\section{The integers $N_{m}$ ARe not the Closest to $\Phi_{m} / 2 \pi$}

In this section, we prove the following result:

Theorem 7.1. There exists an embedded planar graph $M$ and a set of data $A_{j}$ on the edges of $M$, such that the minimizers of $\mathcal{H}$ do not satisfy for all cycle $l$ bordering the regions $F_{l}$ the condition

$$
\left|N_{l}-\Phi_{l} / 2 \pi\right| \leq 1 / 2
$$

Proof. The proof is based on Corollary [3.2, which states that the solution of the minimization problem for $\mathcal{H}$ does not see how the graph $M$ is embedded in the plane. It sees only the data $A$.

We argue by contradiction: assume that for all graphs $M$ and all sets of data $A_{j}$, relation (7.1) holds, and let us pick a particular graph, as sketched in Fig. 1 .

To make things more precise, we assume that the length of all the edges of $M$ is equal to $L$, we let $a_{j}=0$ and $b_{j}=L$ for all $j=1, \ldots, 4$ and we select data $A_{j}$ such that

$$
\int_{0}^{L} A_{1} d \theta=\frac{6 \pi}{5}, \quad \int_{0}^{L} A_{2} d \theta=\frac{2 \pi}{5}, \quad \int_{0}^{L} A_{3} d \theta=-\frac{2 \pi}{5}, \quad \int_{0}^{L} A_{4} d \theta=-\frac{6 \pi}{5} .
$$

On the oriented regions enclosed by the graph, we have

$$
\Phi_{1}=\Phi_{2}=\Phi_{3}=\frac{4 \pi}{5} .
$$

Therefore, for any minimizer $f$ of $\mathcal{H}$, the hypothesis implies that

$$
N_{1}=N_{2}=N_{3}=0 \text {. }
$$

In particular, if we consider the cycle $m$ which is the sum of the three basis cycles of the picture, we can see that

$$
N_{m}=0, \quad \frac{\Phi_{m}}{2 \pi}=\frac{6}{5} .
$$


(1)

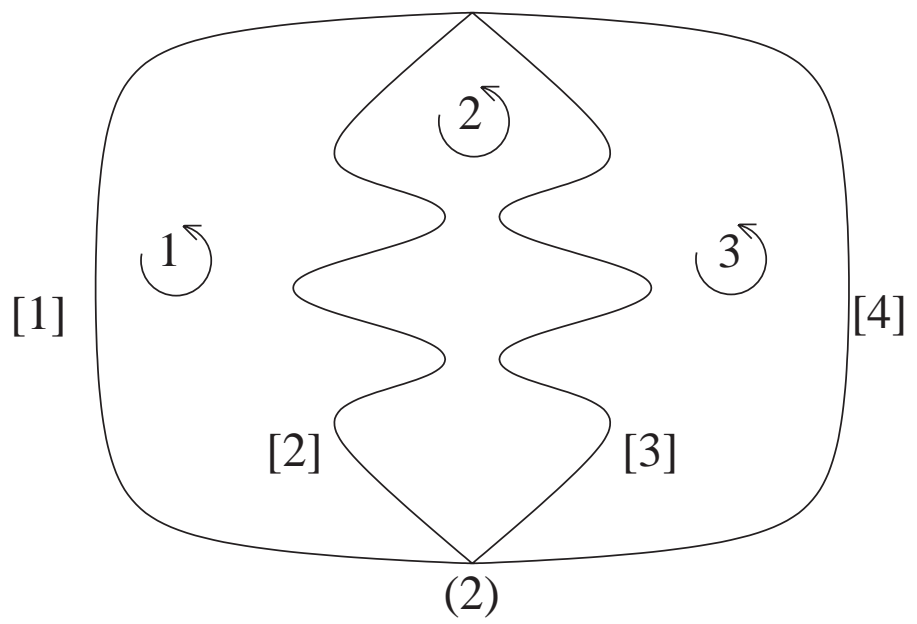

Figure 1. The labels of the vertices are enclosed in parentheses, and the labels of the edges are enclosed in square brackets; the regions are indicated with their orientation.

This does not contradict yet the assumption, since this cycle does not border a connected component of $\mathbb{R}^{2} \backslash M$.

Let us now define new data $\widehat{A}_{j}$ by

$$
\widehat{A}_{1}=A_{4}, \quad \widehat{A}_{2}=A_{1}, \quad \widehat{A}_{3}=A_{2}, \quad \widehat{A}_{4}=A_{3} .
$$

Then any minimizer of $\mathcal{H}$ with the hatted data is obtained by circular permutation from a minimizer of $\mathcal{H}$ with non-hatted data: if $\left(f_{j}\right)_{1 \leq j \leq 4}$ is a minimizer of $\mathcal{H}$ with non-hatted data, then a minimizer of $\mathcal{H}$ with hatted data is given by

$$
\widehat{f}_{1}=f_{4}, \quad \widehat{f}_{2}=f_{1}, \quad \widehat{f}_{3}=f_{2}, \quad \widehat{f}_{4}=f_{3},
$$

since the functional is identical, up to circular permutation. In particular, $\widehat{\phi}_{j}$ can be taken as the circular permutation of $\phi_{j}$. But, we can calculate $\widehat{N}_{1}$ as follows:

$$
\begin{aligned}
2 \pi \widehat{N}_{1} & =\widehat{\phi}_{1}(L)-\widehat{\phi}_{1}(0)-\widehat{\phi}_{4}(L)+\widehat{\phi}_{4}(0) \\
& =\phi_{4}(L)-\phi_{4}(0)-\phi_{1}(L)+\phi_{1}(0) \\
& =2 \pi\left(N_{1}+N_{2}+N_{3}\right) \\
& =0 .
\end{aligned}
$$

On the other hand, we may calculate the circulation of $\widehat{A}$ on each of the cycles; in particular

$$
\widehat{\Phi}_{1}=-\frac{12 \pi}{5}
$$

This contradicts the initial assumption, since $\widehat{N}_{1}$ vanishes. 


\section{ACKNOWLEDGMENT}

It is a pleasure to thank here Ron Aharoni for providing an observation which triggered the proof of Theorem 7.1 .

\section{REFERENCES}

[1] H.J. Carlin and A.B. Giordano. Network Theory. Prentice Hall, 1964.

[2] Y.Colin de Verdière, Y.Pan, and B.Ycart. Singular limits of Schrödinger operators and Markov processes. J. Operator Theory, 41(1):151-173, 1999. MR 2000e:47031

[3] W.A. Little and R.D. Parks. Observation of quantum periodicity in the transition temperature of a superconducting cylinder. Phys. Rev. Lett., 9:9-12, 1962.

[4] V.V. Moshchalkov, L.Gielen, and Y.Bruynseraede. Effect of sample topology on the critical fields of mesoscopic superconductors. Nature, 373(6512):319, 1995.

[5] B.Pannetier. Superconducting wire networks. In B.Kramer, editor, Quantum Coherence in Mesoscopic Systems, pages 457-484. Plenum Press, 1991.

[6] J.Rubinstein and M.Schatzman. Variational problems on multiply connected thin strips IV: Zero sets for the Ginzburg-Landau linearized equations. in preparation.

[7] J.Rubinstein and M.Schatzman. Variational problems on multiply connected thin strips II: convergence of the Ginzburg - Landau functional. Technical Report 294, UMR 5585 CNRS Equipe d'Analyse Numérique, April 1999.

Department of Mathematics, Technion, Haifa 32000, Israel

E-mail address: koby@leeor.technion.ac.il

UMR 5585 CNRS MAPly, Laboratoire de Mathématiques Appliquées de Lyon, Université Claude Bernard - Lyon 1, 69622 Villeurbanne Cedex, France

E-mail address: schatz@maply.univ-lyon1.fr 